\title{
A Family of Styrylcoumarins: Synthesis, Spectroscopic, Photophysical and Photochemical Properties
}

\author{
João Avó, ${ }^{[a]}$ Sérgio Martins, ${ }^{[b]}$ A. Jorge Parola ${ }^{[a]}$ João C. Lima, ${ }^{[a]}$ Paula S. Branco, ${ }^{*[a]}$ \\ João P. Prates Ramalho, ${ }^{[b]}$ and António Pereira* ${ }^{*[b]}$
}

Photochromism, which is the reversible transformation between two molecular forms with different absorption spectra, resulting from photoirradiation, has applicability in several fields, including light modulation materials, optical recording materials, optical switches and photochromic inks. ${ }^{[1]}$ The switching from one molecular state to another is dependent upon the efficiency of light absorption by the input chromophore and its ability to undergo a photochemical reaction. Coumarins are an old class of compounds that naturally occur in several plant families. Owing to their relevant and diverse pharmacological and biochemical properties, ${ }^{[2]}$ and also owing to numerous studies related to their application in probes and fluorescence sensors, ${ }^{[3]}$ interest in this class of compounds is growing exponentially. Upon substitution with various functional groups at different positions, the coumarin chromophores expand their interesting properties. The usually strong fluorescence in the visible-light range makes them useful in laser dyes and organic light-emitting diodes (LEDs). ${ }^{[4]}$ In recent years, we have devoted some interest to the study of coumarin chromophores, with particular emphasis on their syntheses and photophysical properties. ${ }^{[5]} \mathrm{A}$ major issue in expanding the applications of coumarin derivatives is related to their colour spectrum and intensity of their spectroscopic bands. One solution arises from increasing the delocalisation of the conjugated $\pi$-electron system; this will allow us to obtain derivatives of coumarins with absorption bands at longer wavelengths and with higher molar extinction coefficients. We have been particularly interested in the extension of the $\pi$-delocalised system of the coumarin chromophore at the 3-position. Our approach involved 1) building of the 3 -vinyl coumarin ring from readily available substrates ${ }^{[5 d]}$ or 2) vinylation of 3-bromocoumarin derivatives. ${ }^{[5 a, b]}$ These 3 -vinyl coumarins were used as a substrate for the synthesis of several 3-styrylcoumarins by very simple and efficient palladium coupling reactions. Depending upon the substitution pattern, the compounds showed pronounced bathochromic shifts and hyperchromic effects. ${ }^{[5 a, b, d]}$ The palladium-catalysed insertion of 3-bromocoumarin into a number of

[a] J. Avó, Prof. Dr. A. J. Parola, Prof. Dr. J. C. Lima, Prof. Dr. P. S. Branco REQUIMTE, Departamento de Quimica Faculdade de Ciências e Tecnologia, FCT Universidade Nova de Lisboa, 2829-516 Caparica (Portugal) E-mail: paula.branco@fct.unl.pt

[b] S. Martins, Prof. Dr. J. P. Prates Ramalho, Prof. Dr. A. Pereira Centro de Química, Departamento de Química Rua Romão Ramalho, 59, 7000-671 Évora (Portugal) E-mail:amlp@uevora.pt

Supporting information for this article is available on the WWW under http://dx.doi.org/10.1002/cplu.201300118. alkenes, cycloalkenes and alkynes was also reported by others. ${ }^{[6]}$ We had the advantage basing our chromophore on 6,7-dihydroxycoumarin (Esculetin), which is a naturally occurring member of the coumarin family. ${ }^{[7]}$ Herein, we propose studying the effect of the extension of the $\pi$-delocalised system of the 6-methoxycoumarin chromophore at the 5-position. The experimental photophysical and -chemical properties of this new family are correlated with computational studies.

The bromination of 6-methoxycoumarin (1) with Oxone $\mathrm{e}^{[5 \mathrm{~b}, 8]}$ and $\mathrm{HBr}$, as previously reported, ${ }^{[5 \mathrm{~b}]}$ led to 5-bromo-6-methoxycoumarin (2) instead of bromination usually observed at the 3position for other coumarin derivatives. Subsequent Suzuki cross-coupling reaction of $\mathbf{2}$ with potassium vinyltrifluoroborate $^{[5 b, 9]}$ allowed 6-methoxy-5-vinylcoumarin (3) to be obtained in very good yield (92\%). Although compound 3 was previously reported by Harayama and Nishita, ${ }^{[10]}$ they did not report any physical data concerning characterisation of the compound. The extension of the $\pi$-electron system to 5 -styrylcoumarins (4) was achieved by Heck palladium coupling reactions in moderate to high yields (Scheme 1 and Table 1) and, as expected, the yield decreased if electron-donating groups were present at the iodoaryl substrate as a consequence of a less favourable palladium insertion step ( $\mathbf{4} \mathbf{b}$ vs. $\mathbf{4 a}$ ). As opposed to the previously reported 3-styrylcoumarins, ${ }^{[5 \mathrm{~d}]}$ the 5 -styrylcoumarin derivatives 4 were obtained as a mixture of both $E$ and $Z$ isomers (Table 1 ).

The photophysics of the synthesised coumarins was studied, and their absorption and emission properties, as well as fluorescence quantum yield $\left(\Phi_{\mathrm{F}}\right)$, excited-state decay lifetimes $(\tau)$, and radiative and non-radiative rate constants $\left(k_{\mathrm{r}}\right.$ and $k_{\mathrm{n} r}$ respectively) are summarised in Table 1 (more details are given in the Supporting Information). The absorption and emission maxima of the more conjugated 5-styryl derivatives ( $4 \mathbf{a}-\mathbf{c})$ are all redshifted relative to unsubstituted coumarin 1 or vinyl derivative 3, in accordance with previous studies on 3-styrylcoumarins. ${ }^{[5 \mathrm{~d}]}$ The extension of the conjugated system in the 5-position increases both the fluorescence quantum yield and excited-state lifetime; thus, essentially affecting the long-lived excited state of these coumarins, behaviour contrary to that exhibited by 3-styryl derivatives, for which more extended conjugation led to a decrease in both photophysical parameters. ${ }^{[5 d]}$ The short fluorescence lifetime component $\left(\tau_{1}\right)$ amplitude (amp) and non-radiative rate constants $\left(k_{\mathrm{nr}}\right)$ are significantly larger for coumarins $\mathbf{1}$ and $\mathbf{3}$; this suggests that 5-styrylcoumarins exhibit different, less efficient non-radiative deactivation pathways (Table 1).

The photochemistry of all coumarins was then investigated. NMR spectroscopy data revealed that, with the exception of $\mathbf{3}$, 


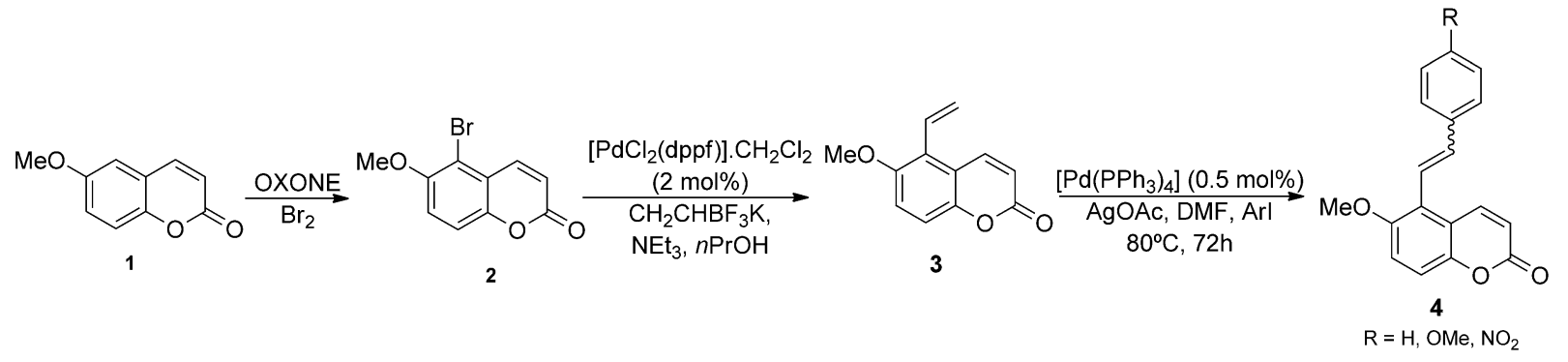

Scheme 1. Synthesis of 6-methoxy-5-styrylcoumarins $4 . \mathrm{DMF}=N, N$-dimethylformamide, $d p p f=1,1^{\prime}$-bis(diphenylphosphino)ferrocene.

\begin{tabular}{|c|c|c|c|c|c|c|c|c|c|c|c|c|c|c|}
\hline Compound & $\mathrm{R}$ & $\begin{array}{l}\text { Yield }^{[a]} \\
{[\%]}\end{array}$ & $Z / E$ ratio $^{[\mathrm{b}]}$ & $\begin{array}{l}\lambda_{\text {abs }} \\
{[\mathrm{nm}]}\end{array}$ & $\begin{array}{l}\varepsilon \\
{\left[\mathrm{cm}^{-1} \mathrm{M}^{-1}\right]}\end{array}$ & $\begin{array}{l}\lambda_{\mathrm{em}} \\
{[\mathrm{nm}]}\end{array}$ & $\Phi_{\mathrm{F}}$ & $\begin{array}{l}\tau_{\mathrm{m}} \\
{[\mathrm{ns}]}\end{array}$ & $\begin{array}{l}\tau_{1} \text { [ns] (amp) } \\
\tau_{2} \text { [ns] (amp) }\end{array}$ & $\begin{array}{l}k_{\mathrm{r}} \\
{\left[\mathrm{ns}^{-1}\right]}\end{array}$ & $\begin{array}{l}k_{\mathrm{nr}} \\
{\left[\mathrm{ns}^{-1}\right]}\end{array}$ & $Z / E^{[c]}$ ratio & $\Phi_{\mathrm{P}}^{[b]}$ & $\begin{array}{l}k_{\mathrm{p}} \\
{\left[\mathrm{ns}^{-1}\right]}\end{array}$ \\
\hline 1 & - & - & - & $\begin{array}{l}275 \\
340\end{array}$ & $\begin{array}{l}12890 \\
4974\end{array}$ & 430 & 0.02 & 0.3 & $\begin{array}{l}0.2(0.89) \\
2.5(0.11)\end{array}$ & 0.06 & 3.06 & - & 0.32 & 1.00 \\
\hline 3 & - & 92 & - & $\begin{array}{l}287 \\
350\end{array}$ & $\begin{array}{l}13619 \\
3128\end{array}$ & 462 & 0.03 & 0.8 & $\begin{array}{l}0.1(0.36) \\
1.1(0.64)\end{array}$ & 0.04 & 1.21 & - & - & - \\
\hline $4 a$ & H & 80 & $32: 68$ & $\begin{array}{l}269 \\
308 \\
360\end{array}$ & $\begin{array}{r}19321 \\
21204 \\
6248\end{array}$ & 512 & 0.12 & 3.2 & $\begin{array}{l}0.8(0.08) \\
3.5(0.92)\end{array}$ & 0.04 & 0.27 & $55: 45$ & 0.59 & 0.18 \\
\hline $4 b$ & OMe & 50 & $33: 67$ & $\begin{array}{l}279 \\
317 \\
370\end{array}$ & $\begin{array}{r}22619 \\
21689 \\
8363\end{array}$ & 576 & 0.30 & 3.9 & $\begin{array}{l}0.1(0.08) \\
4.2(0.92)\end{array}$ & 0.08 & 0.18 & 45:55 & 0.44 & 0.11 \\
\hline $4 c$ & $\mathrm{NO}_{2}$ & 90 & $17: 83$ & 333 & 22268 & 475 & $<0.01$ & $n / a$ & $\mathrm{n} / \mathrm{a}$ & $\mathrm{n} / \mathrm{a}$ & $n / a$ & 49:51 & 0.75 & $\mathrm{n} / \mathrm{a}$ \\
\hline
\end{tabular}

[a] Yield of product isolated. [b] Based on NMR spectroscopy data. [c] Photostationary state, determined from NMR spectroscopy data. $\mathrm{n} / \mathrm{a}=$ not available.

all coumarins undergo photochemical reactions upon irradiation at appropriate wavelengths. However, there were two distinct photochemical reactions occurring in these coumarins. Although 1 underwent photodimerisation with a quantum yield of 0.32 (NMR spectra are given in the Supporting Information), 5 -styrylcoumarins $\mathbf{4 a}, \mathbf{4 b}$ and $\mathbf{4 c}$ exhibited $E-Z$ isomerisation around the exocyclic $\mathrm{C}_{\alpha}=\mathrm{C}_{\beta}$ bond with relatively high quantum yields (from 0.44 to 0.75 ). On the other hand, irradiation of 3 did not give rise to any changes in the NMR or UV/Vis absorption spectra. This shows that $\mathbf{3}$ does not undergo photodimerisation and the radiationless deactivation pathway could be similar to that of the 5-styryl derivatives, which goes through rotation around the exocyclic $\mathrm{C}_{\alpha}=\mathrm{C}_{\beta}$ bond upon irradiation. This hypothesis is compatible with the observed absence of changes in the NMR spectra because, in compound $\mathbf{3}$, there are no substituents at the $\beta$ position. Computational studies ${ }^{[11]}$ show that the exocyclic double-bond length of styrene increases in the first excited state, reducing the double-bond character and facilitating rotation. Therefore, a similar non-radiative deactivation of the excited state can take place in $\mathbf{3}$. To support this hypothesis, similar computational studies were conducted for coumarins $\mathbf{3}$ and $\mathbf{4 a}$. Frontier molecular orbitals for these compounds are shown in Figure 1. For coumarins $\mathbf{3}$ and 4, the $S_{1}$ state is mainly due to the HOMO-LUMO transition, both for the $E$ and $Z$ isomers. Excitation of the most abundant
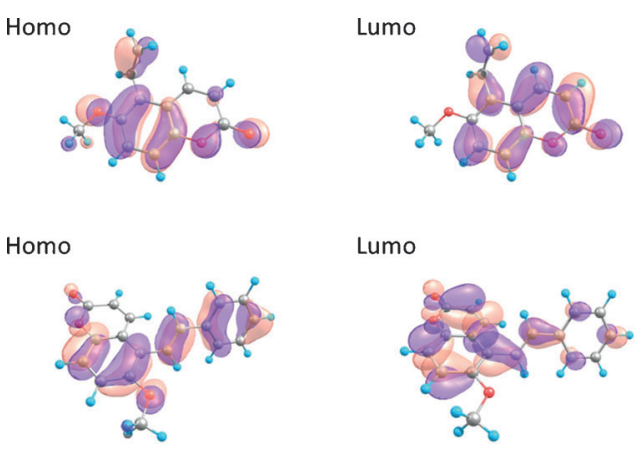

Figure 1. Frontier orbitals for compounds $\mathbf{3}$ (top) and (E)-4a (bottom) obtained from density functional theory (DFT) calculations.

$E$ isomer to form the first excited state leads to a change in the molecular orbital symmetry around $C_{\alpha}-C_{\beta}$. Thus, electron excitation from the HOMO to the LUMO leads to a reduction in the electronic density around the $\mathrm{C}=\mathrm{C}$ bond, and therefore, to a decrease in the rotational barrier for the excited state, for both $\mathbf{3}$ and $\mathbf{4} \mathrm{a}$. These results suggest that the main deactivation pathway for $\mathbf{3}$ is similar to that of 5 -styrylcoumarins, that is, rotation around the $\mathrm{C}=\mathrm{C}$ bond, and thus, the photodimerisation pathway does not occur in coumarins with vinyl or styryl 
substituents at the 5-position. The fact that the value of $k_{\mathrm{nr}}$ is significantly larger for 3, compared with 5-styrylcoumarins, could arise from the lack of substituents at $C_{\beta}$, which may slow rotation around the $\mathrm{C}=\mathrm{C}$ bond.

The relatively high isomerisation quantum yields obtained for $\mathbf{4 a}$ and $\mathbf{4 b}$ justify their low fluorescence quantum yields, compared with their 3-styryl counterparts. ${ }^{[5 \mathrm{~d}]}$ Coumarin $4 \mathrm{c}$ has a very low fluorescence quantum yield, as expected from previous studies on coumarins with nitro moieties. ${ }^{[5 \mathrm{~d}]}$ From UV and NMR spectroscopy results, it was determined that samples from 5-stryrylcoumarins 4 had both $E$ and $Z$ isomers, even before irradiation (Table 1); this indicates that both isomers are formed during the final Heck reaction and suggests that the energy gap between the $E$ and $Z$ species is small. The energy differences between the $E$ and $Z$ isomers for compounds $4 \mathrm{a}$, $\mathbf{4 b}$ and $4 \mathrm{c}$ were determined by DFT calculations with different basis sets and the polarisable continuum model (PCM) for methanol as the solvent (details are given in the Supporting Information). In all cases, an energy difference between the $E$ and $Z$ isomers was found and the $E$ isomer had a lower energy. The inclusion of zero-point energy (ZPE) corrections slightly reduced the energy differences but did not change the trend. If a larger base (B3LYP/aug-cc-pVDZ) was used, the energy difference between the isomers was reduced and further inclusion of solvent slightly increased these energy differences, resulting in the biggest energy difference between the two isomers for 4b. Nevertheless, the $E$ isomer is the most stable form in all cases. These results are in agreement with the NMR spectroscopy data and show that the $E$ isomer is the most abundant form after synthesis and purification steps for all compounds 4 (Table 1).

To evaluate the applicability of these 5-styrylcoumarin derivatives as photochromic molecules, $E-Z$ isomerisation was also followed by UV/Vis spectroscopy. Table 1 shows the initial and photostationary state molar fractions of both isomers, as well as the photoreaction quantum yields $\left(\Phi_{\mathrm{p}}\right.$; dimerisation for 1 and isomerisation for $4 \mathrm{a}-\mathrm{c}$ ) and respective rate constants $\left(k_{\mathrm{p}}\right)$. Spectral changes observed upon irradiation of a solution of $\mathbf{4 a}$ in methanol are depicted in Figure 2. Because all compounds were isolated as a mixture of isomers, irradiation was performed at the lowest energy transition to irradiate the most abundant $E$ isomer preferentially. The decrease in absorbance at the absorption maximum of $\lambda=308 \mathrm{~nm}$, concomitant with an increase in the absorbance at the lower wavelength part of the spectrum, is consistent with $E-Z$ photoisomerisation; the $Z$ isomer is less stable owing to steric hindrance that forces the aromatic ring out of the plane and prevents more extended conjugation. The relatively large $k_{\mathrm{p}}$ values calculated for $4 \mathrm{a}$ and $\mathbf{4} \mathbf{b}$ indicate that photoisomerisation is the main non-radiative pathway of the deactivation of the excited state for 5-styrylcoumarins and justify the low fluorescence of these derivatives. The difference in the isomerisation quantum yields between coumarins $\mathbf{4 a}, \mathbf{4 b}$ and $\mathbf{4 c}$ can be justified by the nature of the substituent at the para position of the styryl group. In principle, electron-donating groups, such as the methoxy group, should increase the double-bond character of the exocyclic double bond, rendering isomerisation less effective,

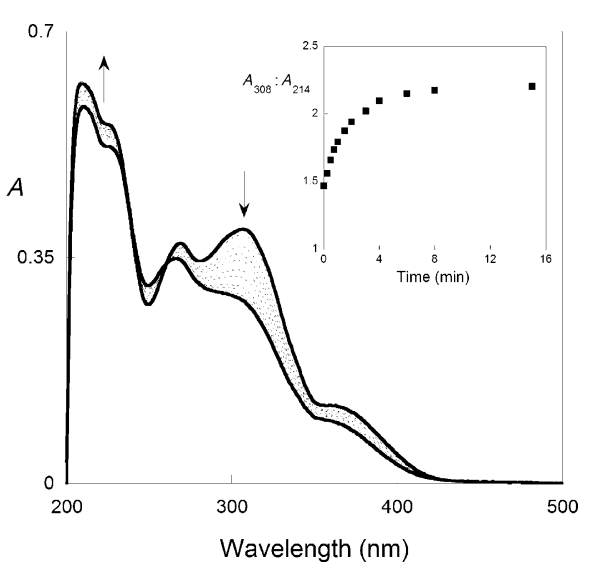

Figure 2. Spectral modification of a solution of 4 a $\left(2.0 \times 10^{-5} \mathrm{M}\right)$ in $\mathrm{MeOH}$, upon irradiation at $\lambda=360 \mathrm{~nm}$. Inset: variation of the absorbance ratio at two different wavelengths, $\lambda=214$ and $308 \mathrm{~nm}$.

whereas electron-withdrawing groups, such as the nitro moiety, should have the opposite effect.

The isomer ratio at the photostationary state was determined by NMR spectroscopy to be about $50 \%$ for $\mathbf{4 a}, \mathbf{4 b}$ and 4c (Table 1). Further conversion was not possible, probably owing to the rather low difference in molar absorptivities of both isomers at the irradiation wavelengths $(\Delta \varepsilon=1143$ for $4 \mathrm{a}$ $(\lambda=317), 703$ for $\mathbf{4 b}(317), 1502 \mathrm{~cm}^{-1} \mathrm{M}^{-1}$ for $\left.\mathbf{4 c}(333 \mathrm{~nm})\right)$. To support this hypothesis, time-dependent density functional theory (TDDFT) calculations for the lowest-lying excited singlet states were conducted for compound $\mathbf{3}$ (see details in the Supporting Information). Figure 3 depicts an overlay of the calculated electronic spectra of both $E$ and $Z$ isomers, as well as a mixture of the $E$ and $Z$ spectra with the experimentally (NMR spectroscopy) determined percentages of 0.68 and 0.32 , respectively (Table 1 ). From this curve, maxima at $\lambda=266,300$ and $335 \mathrm{~nm}$ can be observed that are consistent with the maxima observed experimentally at $\lambda=269,308$ and $360 \mathrm{~nm}$ (Table 1). The observed spectral changes upon irradiation of compound 4 a (Figure 2) can now be justified as a decrease in

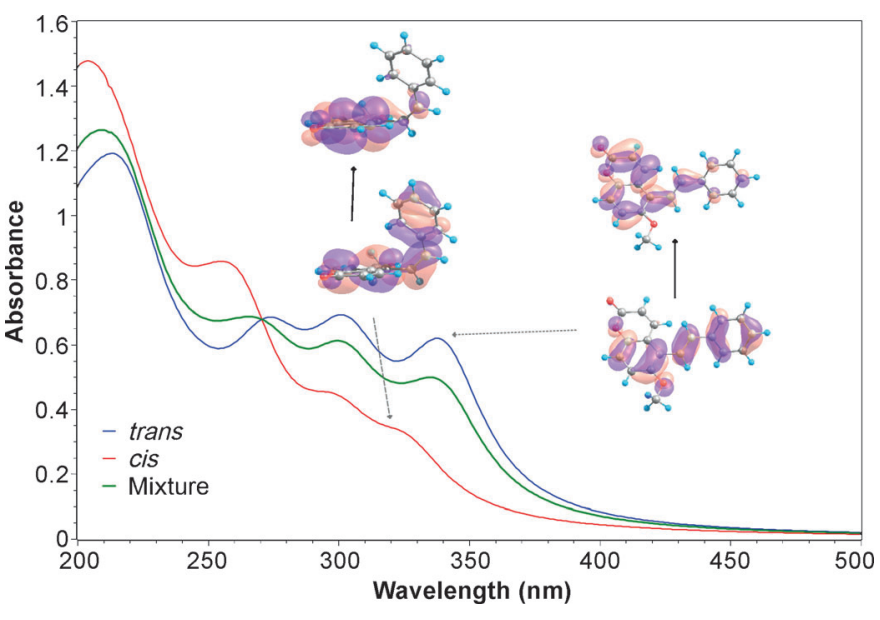

Figure 3. Calculated spectra for the $Z$ and $E$ isomers of $\mathbf{4} \mathbf{a}$ and the combined spectra of the mixture of isomers with experimental molar fractions. 
the amount of $E$ isomer, which is the species that absorbs preferentially in the $\lambda=300 \mathrm{~nm}$ region, and an increase in the $Z$ isomer, which is the isomer that absorbs strongly at higher energies. The photochemistry of coumarins $\mathbf{4 a - c}$ was also tested in plastic media. After adsorption in a thin $\mathrm{N}$-hydroxyethylacrylamide/poly(ethyleneglycol)dimethacrylate copolymer film with coumarin $\mathbf{4} \mathbf{b}$-adsorbed films, these derivatives were irradiated and their photoreactivity was followed by spectrofluorimetry. Visual and spectral modifications of these preliminary results can be found in the Supporting Information.

The pronounced modification of the electronic structure of the new family of 5-styrylcoumarins in relation to the 3-styrylcoumarin family resulted in the alteration of the absorption and emission properties of the chromophore.

Coumarins are usually highly fluorescent molecules that find applications as dyes and fluorescent probes. Extension of the conjugated system of these compounds has been used to yield fluorescent molecules that absorb light at longer wavelengths. ${ }^{[5 d]}$ However, for the compounds described herein, extension of the conjugated system at the 5-position yielded a new type of coumarin derivatives with different photophysical and -chemical properties. The introduction of 5-styryl moieties rendered coumarins with low to moderate fluorescence quantum yields. Instead of noteworthy emissive properties, a new photochemical deactivation pathway through $E-Z$ isomerisation around the $C_{\alpha}=C_{\beta}$ bond with reasonable quantum yields was detected both in liquid and solid media and corroborated by computational studies. We anticipate that this behaviour could be explored for the use of these compounds in optical memory devices.

\section{Acknowledgements}

This study has been supported by the Fundação para a Ciência e a Tecnologia through grant no. PEst-C/EQB/LA0006/2011 and FCOMP-01-0124-FEDER-007448. J.A. wishes to thank the FCT for a PhD grant (SFRH/BD/65127/2009). The NMR spectrometers are part of the National NMR Network (RNRMN) and are funded by the Fundação para a Ciência e a Tecnologia (FCT).

Keywords: coumarins • density functional calculations isomerization $\cdot$ photochemistry $\cdot$ photochromism

[1] a) H. Bouas-Laurent, H. Durr, Pure Appl. Chem. 2001, 73, 639-665; b) M. Irie, Chem. Rev. 2000, 100, 1685-1716; c) V. I. Minkin, Chem. Rev. 2004, 104, $2751-2776$.

[2] a) F. Borges, F. Roleira, N. Milhazes, L. Santana, E. Uriarte, Curr. Med. Chem. 2005, 12, 887-916; b) I. Kostova, Curr. Med. Chem. Anti Canc. Agents, 2005, 5, 29-46.

[3] a) H. E. Katerinopoulos, Curr. Pharm. Des. 2004, 10, 3835-3852; b) W. Y. Lin, L. Yuan, X. W. Cao, W. Tan, Y. M. Feng, Eur. J. Org. Chem. 2008, $4981-4987$; c) H. S. Jung, P. S. Kwon, J. W. Lee, J. I. Kim, C. S. Hong, J. W. Kim, S. H. Yan, J. Y. Lee, J. H. Lee, T. Joo, J. S. Kim, J. Am. Chem. Soc. 2009, 131, 2008-2012.

[4] a) C. H. Chang, H. C. Cheng, Y. J. Lu, K. C. Tien, H. W. Lin, C. L. Lin, C. J. Yang, C. C. Wu, Org. Electron. 2010, 11, 247-254; b) T. Z. Yu, P. Zhang, Y. L. Zhao, H. Zhang, J. Meng, D. W. Fan, Org. Electron. 2009, 10, 653660 ; c) M. T. Lee, C. K. Yen, W. P. Yang, H. H. Chen, C. H. Liao, C. H. Tsai, C. H. Chen, Org. Lett. 2004, 6, $1241-1244$.

[5] a) S. Martins, P. S. Branco, M. C. de La Torre, M. A. Sierra, A. Pereira, Synlett 2010, 2918-2922; b) S. M. A. Martins, P. S. Branco, A. M. D. R. L. Pereira, J. Braz. Chem. Soc. 2012, 23, 688-693; c) J. S. Seixas de Melo, C. Cabral, J. C. Lima, A. L. Macanita, J. Phys. Chem. A 2011, 115, 8392 8398; d) J. Gordo, J. Avó, A. J. Parola, J. C. Lima, A. Pereira, P. S. Branco, Org. Lett. 2011, 13, 5112-5115.

[6] a) M. S. Schiedel, C. A. Briehn, P. Bauerle, Angew. Chem. 2001, 113, $4813-$ 4816; Angew. Chem. Int. Ed. 2001, 40, 4677-4680; b) S. Valente, E. Bana, E. Viry, D. Bagrel, G. Kirsch, Bioorg. Med. Chem. Lett. 2010, 20, 58275830.

[7] G. J. Smith, C. L. Dunford, P. B. Roberts, J. Photochem. Photobiol. A 2010, $210,31-35$.

[8] K. M. Kim, I. H. Park, Synthesis 2004, 2641-2644.

[9] G. A. Molander, R. Figueroa, Aldrichimica Acta 2005, 38, 49-56.

[10] T. Harayama, Y. Nishita, Chem. Pharm. Bull. 1996, 44, 1986-1988.

[11] a) J. Del Nero, C. P. De Melo, Int. J. Quantum Chem. 2003, 95, 213-218; b) J. M. Smith, J. L. Knee, Laser Chem. 1994, 14, 131-141.

Received: April 3, 2013

Published online on June 20, 2013 